Radiologe 2021 $61: 703$

https://doi.org/10.1007/s00117-021-00889-1

Angenommen: 24. Juni 2021

(c) Springer Medizin Verlag $\mathrm{GmbH}$, ein Teil von Springer Nature 2021

Liebe Leserinnen und Leser,

Verletzungen der Halswirbelsäule (HWS) treten bei Traumata auf, werden aber oft übersehen oder spät diagnostiziert. Ein Schleudertrauma ist eine relativ häufige Verletzung der Halswirbelsäule, die meistens im Rahmen von Verkehrsunfällen (in der Regel mit einem Heckaufprall) beobachtet wird. Typisch hierfür ist eine plötzliche peitschenartige Kopf- und Halsbewegung (Reklination gefolgt von Inklination), die zur Verletzung der zervikalen Weichteile, Bänder und Knochen führen kann. Zu den häufigsten Symptomen gehören zervikale Schmerzen, Nackensteifigkeit und -überempfindlichkeit, die mit einer Latenz von Stunden auftreten; die Beschwerden können bei einigen Patienten einen chronischen Verlauf annehmen. In der bildgebenden Diagnostik werden selten traumaassoziierte Veränderungen nachgewiesen; es gibt außerdem keine spezifischen Befunde, die für ein Schleudertrauma typisch sind. Der Kopf macht etwa $8 \%$ des Körpergewichts eines Erwachsenen aus, wird jedoch nur durch die Halswirbelsäule und relativ schwache Ligamente stabilisiert. Bei Kindern ist dieses Verhältnis sogar noch ungünstiger. In diesem Artikel werden die verschiedenen Klassifikationen der Wirbelsäulenverletzungen auf-

\section{Autor}

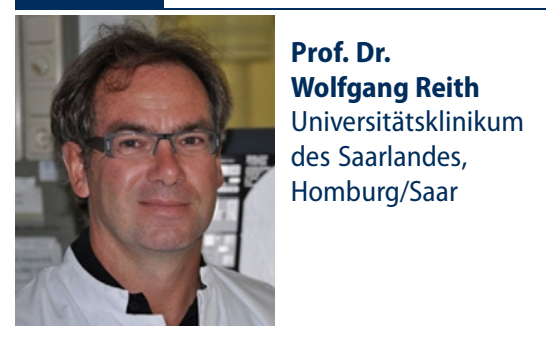

\title{
Diagnostik von Traumata der Halswirbelsäule
}

\author{
Wolfgang Reith · Umut Yilmaz
}

Klinik für Diagnostische und Interventionelle Neuroradiologie, Universitätsklinikum des Saarlandes, Homburg/Saar, Deutschland

gezeigt. Bei Rasanztraumata muss neben den klassischen Frakturen eine Verletzung der A. vertebralis ausgeschlossen werden.

Dissektionen der hirnversorgenden Arterien entstehen traumatisch, iatrogen und spontan ohne sichere Auslöser. Letztere gehören zu den häufigsten Ursachen sog. juveniler Schlaganfälle, also solchen, die in einem Lebensalter von unter 55 Jahren auftreten.

Neben der Diagnostik werden auch die Indikationen zu neurochirurgischen Eingriffen bei Traumata erörtert. Bei Schädelbasisfrakturen handelt es sich um Frakturen des unteren Abschnitts des Hirnschädels. Sie machen ca. 20\% aller Schädelfrakturen aus und werden vor allem durch Hochranztraumata und Stürze aus großer Höhe verursacht. Sie können in Abhängigkeit ihrer genauen Lokalisation in frontobasale, laterobasale und frontolaterale Frakturen unterteilt werden. Mögliche klinische Zeichen sind das Vorliegen einer Rhino- und Otoliquorrhoe, Monokelund Brillenhämatome, retroaurikuläre Ekchymose (sog. Battle-Zeichen) sowie Hirnnervenausfälle.

Nicht zu unterschätzen und häufig übersehen werden nichtakzidentelle Schädel-Hirn-Traumata (SHT) im Kindesalter. Akzidentelle und nichtakzidentelle Traumata sind nach wie vor weltweit die häufigste Todesursache im Kindesalter. Es wird davon ausgegangen, dass ca. $20 \%$ der SHT bei Kindern unter 2 Jahren nichtakzidentell verursacht sind. Das nichtakzidentelle SHT wird durch gewaltsame Einwirkung auf das Gehirn und Rückenmark verursacht, hervorgerufen durch das massive Hin- und Herschütteln des an den Oberarmen bzw. Körper festgehaltenen Kindes. Dies kann zu einem Abriss von Blutgefäßen, insbesondere von Brückenvenen, führen sowie zu axonalen Scherverletzungen der Nervenverbindungen und Hirnschwellung. Durch die Mitbeteiligung des Hirnstamms kann es zu einem initialen kurzfristigen Atemstillstand kommen.

Verletzungen der HWS, vor allem durch Traumata, sind häufig und benötigen für die weitere Therapie eine umfassende Diagnostik.

Ihre

\section{Wolfgang Reith}

Umut Yilmaz

\section{Korrespondenzadresse}

Prof. Dr. Wolfgang Reith

Klinik für Diagnostische und Interventionelle Neuroradiologie, Universitätsklinikum des Saarlandes

Kirrberger Straße, 66424 Homburg/Saar, Deutschland

Wolfgang.Reith@uniklinikum-saarland.de

Interessenkonflikt. W. Reith und U. Yilmaz geben an, dass kein Interessenkonflikt besteht. 